\title{
Conductivity tensor of striped quantum Hall phases
}

\author{
Felix von Oppen ${ }^{1}$, Bertrand I. Halperin ${ }^{2}$, and Ady Stern ${ }^{3}$ \\ ${ }^{1}$ Institut für Theoretische Physik, Universität zu Köln, Zülpicher Str. 77, 50937 Köln, Germany \\ ${ }^{2}$ Physics Department, Harvard University, Cambridge, Massachusetts, 02139 \\ ${ }^{3}$ Department of Condensed Matter Physics, The Weizmann Institute of Science, 76100 Rehovot, Israel
}

(April 7, 2018)

We study the transport properties of pinned striped quantum Hall phases. We show that under quite general assumptions, the macroscopic conductivity tensor satisfies a semicircle law. In particular, this result is valid for both smectic and nematic stripe phases, independent of the presence of topological and orientational defects such as dislocations and grain boundaries. As a special case, our results explain the experimental validity of a product rule for the dissipative part of the resistivity tensor, which was previously derived by MacDonald and Fisher for a perfect stripe structure.

PACS numbers: 73.40.Hm, 73.20.Dx, 72.10-d

Recent experiments [1,2] have revealed strikingly anisotropic $d c$ transport properties of very clean two dimensional (2D) electron systems when the Landau level filling factor is close to $\nu=N+1 / 2$, and $N \geq 4$ is an integer. It is believed that this is related to previous theoretical proposals [3, 国 that Coulomb interactions would lead to an instability towards charge-density wave (CDW) formation in high, spin resolved, Landau levels (LL). Specifically, formation of a striped phase of the uppermost Landau level was predicted when it is close to half filling, while a "bubble phase" should be favorable further away from half filling. The striped phase consists of one-dimensional stripes alternating between the integer filling factors $N$ and $N+1$ with period of order of the cyclotron radius $R_{c}$. In the bubble phase, clusters of minority filling factor with size $R_{c}$ order in a triangular lattice. These predictions, obtained within the HartreeFock (HF) approximation, have also been supported by numerical exact diagonalization studies [5].

At finite temperature and/or in the presence of disorder, the perfect stripe ordering predicted by HF calculations will presumably be destroyed. The unidirectional CDW shares the symmetries of $2 \mathrm{D}$ smectic liquid crystals [6]. This implies that if there is no external force tending to align the stripes, then a dislocation will cost only a finite amount of energy and thus there will be a finite density of dislocations at non-zero temperatures. This is expected to destroy translational long-range order, except at zero temperature, but preserve quasi-longrange orientational order of the remaining stripe segments, characteristic of a $2 \mathrm{D}$ nematic phase. The orientational order would be effectively locked in by any small added anisotropy. As the temperature becomes large enough, there will be a Kosterlitz-Thouless transition to an isotropic state in which the stripe segments lose their orientational order. Short-range stripe order should disappear completely only around the presumably much higher HF transition temperature.

Transport properties of the striped phases should be affected by even small amounts of disorder on the substrate, which will pin the stripe positions at low temperatures. Disorder should also lead to a finite density of dislocations, even at zero temperature. Moreover, since the forces aligning the stripes are believed to be very weak, steps or other large-scale features of the GaAs-AlGaAs interface may lead to large regions where the stripes are oriented differently from the average preferred direction.

In the present paper, we focus on the transport properties of general striped quantum Hall phases, allowing in particular for the presence of topological defects such as dislocations and grain boundaries. Assuming that the defects are pinned by disorder, we find under quite general assumptions (specified below) that the macroscopic conductivity tensor $\hat{\sigma}^{*}$ satisfies the semicircle law

$$
\sigma_{1}^{*} \sigma_{2}^{*}+\left(\sigma_{h}^{*}-\sigma_{h}^{0}\right)^{2}=\left(e^{2} / 2 h\right)^{2},
$$

with $\sigma_{h}^{0}=(N+1 / 2) e^{2} / h$. Here, we decomposed the macroscopic conductivity $\hat{\sigma}^{*}=\hat{\sigma}_{d}^{*}+\sigma_{h}^{*} \hat{\epsilon}$ into its dissipative part $\hat{\sigma}_{d}^{*}$ and Hall component $\sigma_{h}^{*} \hat{\epsilon}$ with $\hat{\epsilon}$ the totally antisymmetric tensor. The dissipative part is a real symmetric matrix with eigenvalues $\sigma_{1}^{*}$ and $\sigma_{2}^{*}$. Our derivation of (11) uses results obtained by Shimshoni and Auerbach [7] for a model of a "quantized Hall insulator".

Following Refs. [3. [- we assume that the system is made up of regions (stripes or stripe segments) of filling factors $N$ and $N+1$, whose positions are fixed in space. The $N$ completely filled Landau levels contribute to the conductivity tensor the pure Hall response $N\left(e^{2} / h\right) \hat{\epsilon}$. The contribution of the $(N+1)$ th LL is due to the chiral edge modes at the boundaries of each electron stripe (stripe of filling factor $N+1$ ): transport occurs by motion along the edge modes and by impurity scattering between them. We assume that scattering occurs predominantly between nearest-neighbor edges, at rates $1 / \tau_{e}$ and $1 / \tau_{h}$ across electron and hole stripes, respectively. Recently, MacDonald and Fisher (MF) [8] have studied the transport properties of such a system, assuming a uniform, topologically perfect stripe structure, 
with scattering rates $\tau_{e}$ and $\tau_{h}$ that may depend on temperature, but are independent of position. Neglecting quantum interference effects and taking the stripes along the $y$ direction, they find for the conductivity tensor [8]

$$
\begin{aligned}
\sigma_{x x} & =\frac{e^{2}}{h} \frac{a}{v_{F}\left(\tau_{e}+\tau_{h}\right)} \\
\sigma_{y y} & ==\frac{e^{2}}{h} \frac{v_{F}}{a} \frac{\tau_{e} \tau_{h}}{\tau_{e}+\tau_{h}} \\
\sigma_{x y} & =\frac{e^{2}}{h}\left(N+\frac{\tau_{e}}{\tau_{e}+\tau_{h}}\right)
\end{aligned}
$$

with $a$ the period of the CDW and $v_{F}$ the velocity of propagation along the edge channels. MF noticed [8] that at the symmetric point, where $\tau_{e}=\tau_{h}$, this leads to the parameter-free predictions

$$
\begin{aligned}
\sigma_{x x} \sigma_{y y} & =\left(e^{2} / 2 h\right)^{2} \\
\sigma_{x y} & =\frac{e^{2}}{h}(N+1 / 2),
\end{aligned}
$$

independent of the period, Fermi velocity, or scattering rate. If one assumes particle-hole symmetry in the partially full LL, then the symmetric point will occur when the total filling factor is $N+1 / 2$. The relation (3) seems to be in reasonable agreement with the experiment 9 .

In fact, the conductivity tensor (2) satisfies the more general semicircle relation (1), which is valid also away from the symmetric point. In the homogeneous case discussed by MF, the macroscopic conductivity tensor is identical to the local conductivity tensor, and we may write $\sigma_{1}^{*}=\sigma_{x x}, \sigma_{2}^{*}=\sigma_{y y}$, and $\sigma_{h}^{*}=\sigma_{x y}$.

Since, as argued above, the real samples are likely to be quite far from the perfect stripe structure, it may be surprising that the product formula (3) compares so favorably with experiment. Moreover, we deduce from Eq. (2) that for a perfect stripe structure the anisotropy at the symmetric point is $\sigma_{y y} / \sigma_{x x}=\left(v_{F} \tau_{e} / a\right)^{2}$. The experimental anisotropy in the conductivity is about five [1, 10], which would imply that the electrons hop between edges after traveling only a distance of a few cyclotron radii along the edge. For such a situation, quantum interference effects should be important, particularly in view of the fact that the experiments are performed at extremely low temperatures, which could lead to deviations from the product rule (3) and the semicircle law (1) 11].

In the following, we show in two different ways that the semicircle and product rules embodied in (11) are in fact valid for much more general stripe structures. This makes the experimental results consistent with a picture where electrons hop between edges much more rarely, while the anisotropy is reduced by the presence of defects such as dislocations and grain boundaries, which cause the local orientation of the stripes to vary from one place to another. In such a picture, neglecting quantum interference may indeed be justified.

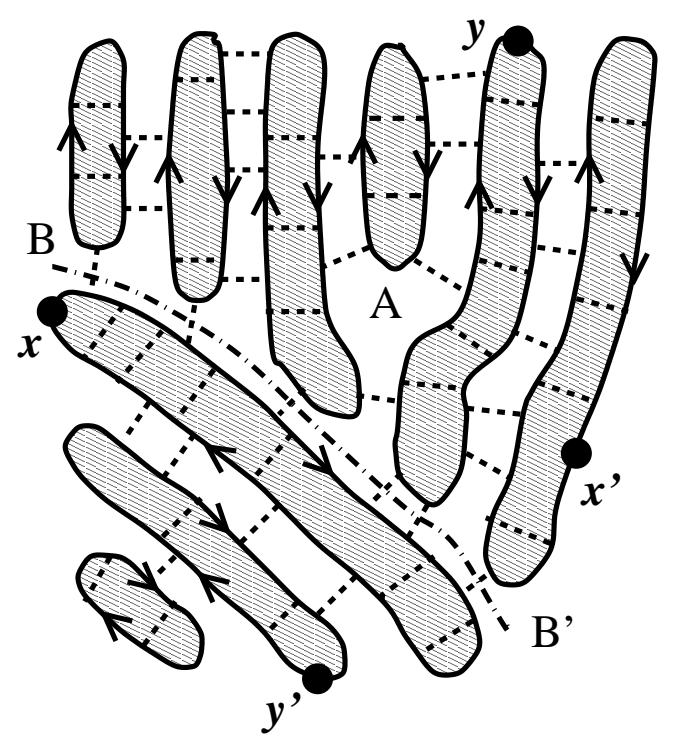

FIG. 1. Small sample of striped phase containing a dislocation $(\mathrm{A})$ and a large angle grain boundary $\left(\mathrm{B}-\mathrm{B}^{\prime}\right)$. Shaded and unshaded regions are incompressible strips with filling factors $N+1$ and $N$ respectively; arrows show direction of electron flow on edge states, for positive magnetic field. Dashed lines represent locations of scattering centers which cause electrons to tunnel between adjacent edge states. Points $x, x^{\prime}, y$ and $y^{\prime}$ are contacts at the edge of the sample.

Consider a general stripe structure such as that shown in Fig. 1. In this figure, shaded and unshaded regions represent incompressible states with filling factors $N+1$ and $N$, respectively. The $N$ filled Landau levels are pure Hall conductors carrying current in parallel to the $(N+$ 1)th LL. We can then focus on the role of the uppermost LL by interpreting the shaded regions in the figure as an incompressible state with $\nu=1$, while the unshaded regions are insulators, with $\nu=0$. At the end, we add the Hall conductance $N e^{2} / h$ of the filled levels.

For the uppermost LL, we now consider a situation where a current $I$ is passed between the contacts at $x$ and $x^{\prime}$ and the voltage $V$ is measured between contacts $y$ and $y^{\prime}$. In general, $V$ has contributions from both the Hall and the dissipative resistivities.

The local current $i$ along a stripe-edge is related to the local chemical potential $\mu$ (measured relative to the uniform equilibrium chemical potential) by

$$
i=\mu \operatorname{sgn}(B) e^{2} / h .
$$

Neglecting quantum interference effects, each scattering center between two adjacent electron stripes, indicated by dashed lines in Fig. 1, can be characterized by a scattering probability $p$. The currents entering and leaving a scattering center, cf. Fig. 2a, are related by current conservation $i_{1}+i_{2}=i_{3}+i_{4}$ and by $i_{3}=(1-p) i_{1}+p i_{2}$ or $i_{4}=(1-p) i_{2}+p i_{1}$. Equivalently, by using (5) we can characterize the scattering center by its resistance $r=\left(h / e^{2}\right)(1-p) / p$, relating the voltage $v=\mu_{2}-\mu_{3}=$ $\mu_{4}-\mu_{1}$ between edges to the current $j=i_{3}-i_{1}$ across. 
In terms of the quantities $j, v$ and $r$, stripe structures such as that shown in Fig. 1 can be viewed as a type of classical resistor network subject to Kirchhoff's laws and Eq. (5) relating currents and voltages on the edges.

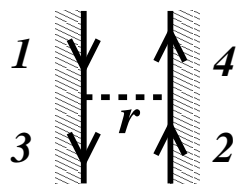

(a)

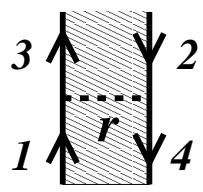

(b)

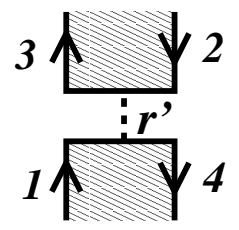

(c)
FIG. 2. Labeling of the current vertices of (a) a scattering center across a hole stripe and (b) across an electron stripe, for $B>0$. The resistances of the scattering centers are denoted by $r$. Any scattering center across an electron stripe as shown in (b) can be replaced by the equivalent dual representation shown in (c) in terms of a scattering center across a hole region with resistance $r^{\prime}$. The resistances of the two junctions are related by $r^{\prime}=\left(h / e^{2}\right)^{2} / r$.

We can "eliminate" the scattering events across electron stripes from the resistor network by using the following dual representation. As indicated in Fig. 2b and 2c, a scattering center across an electron stripe described by $j, v, r$ is equivalent to a scattering center between electron stripes described by $j^{\prime}, v^{\prime}, r^{\prime}$, where $j^{\prime}=i_{4}-i_{1}$, $v^{\prime}=\mu_{2}-\mu_{4}$, and $r^{\prime}=v^{\prime} / j^{\prime}$. This is equivalent to the direct relations $j^{\prime}=\left(e^{2} / h\right) v, v^{\prime}=\left(h / e^{2}\right) j$, and $r^{\prime}=\left(h / e^{2}\right)^{2} / r$ between unprimed and primed parameters. The relation between $r$ and $r^{\prime}$ can be simply understood by noting that Fig. $2 \mathrm{~b}$ maps onto Fig. $2 \mathrm{c}$ when $p$ is replaced by $1-p$.

With this dual representation, the network is defined by a set of puddles (of filling factor $\nu=1$ ) each of which is encircled by one chiral edge mode. Electrons can scatter only between adjacent puddles as described by resistors connecting the puddles. The network is planar in the sense that there are no crossing resistor links between puddles. The Hall voltage $V_{H}$ is even under simultaneous reversal of magnetic field $B$ and current $I$ so that it can be distinguished from the longitudinal voltage by the definition $V_{H} \equiv[V(B, I)+V(-B,-I)] / 2$. By the definition of the network, the effect of changing the sign of $B$ is to reverse the directions of the arrows in the figure, thus changing the sign in (5), while leaving unchanged the values of the resistors $r$ and $r^{\prime}$. This is consistent with the time-reversal properties of the underlying microscopic Hamiltonian.

This resistor network (puddle model) has been studied in the context of the quantized Hall insulator by Shimshoni and Auerbach [7]. These authors prove that when quantum interference between inter-puddle hopping events is neglected the Hall resistance $R_{H}=V_{H} / I=$ $h / e^{2}$. Then, if the network is statistically homogeneous, but not isotropic, and the sample is large compared to any correlation length for fluctuations, we may adapt this result to our problem and define macroscopic conductivity and resistivity tensors for the uppermost LL, $\hat{\sigma}^{u}$ and $\hat{\rho}^{u}$. Let us choose the sample to have a Hall-bar geometry, aligned with the principal axes of $\hat{\rho}^{u}$. Then, using the Onsager symmetry relations, we find

$$
\rho_{y x}^{u}(B)=-\rho_{x y}^{u}(B)=-\rho_{y x}^{u}(-B)=h / e^{2} .
$$

This implies that $\operatorname{det}\left(\hat{\sigma}^{u}\right)=\sigma_{x y}^{u} e^{2} / h$, and the components of $\hat{\sigma}^{u}$ satisfy the semicircle law (1), with $\sigma_{h}^{0}=$ $e^{2} / 2 h$. Finally, if we add the parallel conductivity of the filled Landau levels, the dissipative conductivity is unchanged, but the Hall conductivity is shifted by $N e^{2} / h$, leading to Eq. (11).

We emphasize that the essential assumptions entering the proof are (a) the neglect of quantum interference 11] and (b) the assumption that the network is planar. The importance of the latter assumption can already be understood in the context of the perfect stripe structure. If we violate the assumption by including hopping to nextnearest neighbor stripes, we find that this enhances the conductivity in the $x$ direction while leaving the diffusion constant in the $y$ direction unchanged. Thus, hopping to next-nearest neighbor stripes leads to deviations from, e.g., the product rule (3). Also, the rule will be invalid if the temperature is so high that dislocations are unpinned, and the stripe pattern itself can drift in the presence of an applied electric field. On the other hand, as in the analysis of Shimshoni and Auerbach [7], it is not necessary to assume that the resistances $r$ and $r^{\prime}$ are ohmic, i.e., independent of the magnitude of the current. What is essential is that the voltage across a resistor is reversed when the current and the magnetic field are reversed.

It is possible to give an alternate (continuum) argument for the validity of the semicircle law (1) for $\hat{\sigma}^{*}$. For this argument, we assume that the most important role of defects is to change both the local orientation of the CDW and the local scattering rates between stripe edges. If the defect density is not too high, these changes occur only on scales large compared to the cyclotron radius and we can describe the system by a local conductivity tensor $\hat{\sigma}(\mathbf{r})$ whose principal axes and diagonal conductivities are functions of position but which locally satisfies the semicircle law:

$$
\sigma_{1} \sigma_{2}+\left(\sigma_{h}-\sigma_{h}^{0}\right)^{2}=\left(e^{2} / 2 h\right)^{2} .
$$

We now show, by an argument employing a duality transformation introduced by Dykhne and Ruzin [12], that the resulting macroscopic conductivity tensor satisfies the semicircle law (1).

The microscopic current distribution $\mathbf{J}(\mathbf{r})$ is determined by the equations

$$
\begin{aligned}
\mathbf{J}(\mathbf{r}) & =\hat{\sigma}(\mathbf{r}) \mathbf{E}(\mathbf{r}) \\
\nabla \cdot \mathbf{J}(\mathbf{r}) & =0 \\
\nabla \times \mathbf{E}(\mathbf{r}) & =0 .
\end{aligned}
$$


Then the dual system $\mathbf{J}^{\prime}, \mathbf{E}^{\prime}, \hat{\sigma}^{\prime}$ defined by the transformation

$$
\begin{aligned}
\mathbf{J} & =a \mathbf{J}^{\prime}-b \hat{\epsilon} \mathbf{E}^{\prime} \\
\mathbf{E} & =c \mathbf{E}^{\prime}-d \hat{\epsilon} \mathbf{J}^{\prime}
\end{aligned}
$$

with $a, b, c, d$ arbitrary constants, satisfies the same set of equations with $\hat{\sigma}^{\prime}=[a+d \hat{\sigma} \hat{\epsilon}]^{-1}(c \hat{\sigma}+b \hat{\epsilon})$. The same relation must hold for the corresponding macroscopic conductivities $\hat{\sigma}^{*}$ and $\hat{\sigma}^{* \prime}$, because the macroscopic currents and voltages are just spatial averages of the microscopic currents and voltages.

Following Dykhne and Ruzin 12, we now choose the primed system as the time reverse of the unprimed system, $\sigma_{i j}^{\prime}=\sigma_{j i}$, and solve for $a, b, c, d$ in terms of $\hat{\sigma}$. Choosing $d=1$ without loss of generality, one finds after some tedious but straight-forward algebra that $c=a$ and $b=\sigma_{1} \sigma_{2}+\sigma_{h}^{2}-2 a \sigma_{h}$ with $a$ remaining arbitrary. This defines an allowed duality transformation if $a, b, c, d$ are constants, independent of position. Exploiting the semicircle law (7), we choose $a=c=\sigma_{h}^{0}$, so that $b=\left(e^{2} / 2 h\right)^{2}-\left(\sigma_{h}^{0}\right)^{2}$, independent of position. We can now combine the duality relation between $\hat{\sigma}^{*}$ and $\hat{\sigma}^{* \prime}$ with the fact that the two systems are related by time reversal, $\sigma_{i j}^{* \prime}=\sigma_{j i}^{*}$, and find that the macroscopic conductivity tensor $\hat{\sigma}^{*}$ satisfies the semicircle law (1).

It is an interesting question whether the experimental validity of the product rule is specific to the CDW model involving local stripe ordering with period comparable to the cyclotron radius. For example, one might hypothesize that close to half filling, the system phase-separates, on a scale much larger than the cyclotron radius, into quantized Hall regions with $\nu=N$ and $\nu=N+1$, with only thin boundaries between the regions. If the shapes of the regions are elongated, with a preference for one particular direction in space, one would find an anisotropic macroscopic conductivity tensor which would obey the semicircle law (11) under appropriate conditions. In such a model, however, one obtains very small values of $\sigma_{1}^{*}$ and $\sigma_{2}^{*}$, except very close to the percolation threshold for the two phases. Thus one would expect the transition between Hall plateaus corresponding to $\nu=N$ and $\nu=N+1$ to occur in a very narrow interval of magnetic field, which is contrary to experimental observations in the samples of interest [1,2].

In order to interpret experimental results in terms of an effective macroscopic conductivity tensor $\hat{\sigma}^{*}$, which is anisotropic but spatially uniform, it is important that there be reasonable equilibration between the edge states of the filled LL and the electrons of the partially filled LL, at all points on the sample boundary. If the density profile at the sample edge is sufficiently gradual, however, the spatial separation of the edge states may cause this equilibration to fail at low temperatures. The use of an effective conductivity tensor can be checked, in principle, by comparing samples with different aspect ratios or different contact locations.

The theory presented here does not address a number of issues raised by the experimental observations [1.22. The mechanism which causes the stripes to line up preferentially with a particular axis of the GaAs substrate is not well understood. There is currently no explanation for the observation that the resistance anisotropy has a prominent dependence on whether the Fermi energy is in the lower or upper spin component of a LL. An explanation for the observed non-linearity in the resistivities has been proposed by MF [8], involving quantum fluctuations of the Luttinger-liquid type; however, there are not yet detailed predictions for the full temperature and current dependences which might be compared with experiment.

In summary, we have studied the transport properties of general striped quantum Hall phases. Assuming that the stripes are pinned by disorder and that scattering between stripes can be considered classically, we have established rather generally the validity of a semicircle law (11) for the macroscopic conductivity tensor. Our results provide an explanation for the experimental validity of the product rule (3) which is a special case of (1). It would be very interesting to check if the more general semicircle law (1) is also obeyed in experiment.

We are grateful to J. Eisenstein, B. Huckestein, M. Shayegan, S. Simon, D. Shahar and H. Stormer for helpful discussions. The work was supported in part by SFB 341, NSF grant DMR-94-16910, US-Israel BSF grant 98354, DIP-BMBF grant and the Israeli Academy of Science. Initial phases of the work were carried out at the ITP, Santa Barbara, in August 1998, with support from NSF grant PHY94-07194.

[1] M.P. Lilly, K.B. Cooper, J.P. Eisenstein, L.N. Pfeiffer, and K.W. West, Phys. Rev. Lett. 82, 394 (1999).

[2] R.R. Du, D.C. Tsui, H.L. Stormer, L.N. Pfeiffer, K.W. Baldwin, and K.W. West, Solid State Comm. 109, 389 (1999).

[3] M.M. Fogler, A.A. Koulakov, and B.I. Shklovskii, Phys. Rev. B 54, 1853 (1996); A.A. Koulakov, M.M. Fogler, and B.I. Shklovskii, Phys. Rev. Lett. 76, 499 (1996).

[4] R. Moessner, J.T. Chalker, Phys. Rev. B 54, 5006 (1996).

[5] E.H. Rezayi, F.D.M. Haldane, and K. Yang, Phys. Rev. Lett. 83, 1219 (1999).

[6] E. Fradkin, S.A. Kivelson, Phys. Rev. B 59, 8065 (1999).

[7] E. Shimshoni and A. Auerbach, Phys. Rev. B 55, 9817 (1997).

[8] A.H. MacDonald and M.P.A. Fisher, cond-mat/9907278.

[9] J. Eisenstein, private communication.

[10] S.H. Simon, cond-mat/9903086.

[11] In general, the semicircle law does not apply to a quantum mechanical model, where quantum localization or interference effects may be important. [See L.P. Pryadko 
and A. Auerbach, Phys. Rev. Lett. 82, 1253 (1999)]. However, the size of the deviation is highly modeldependent, and could be small, so one cannot necessarily exclude quantum interference effects on the basis of the experimental results.

[12] A.M. Dykhne, I.M. Ruzin, Phys. Rev. B 50, 2369 (1994). 